DOI: 10.17707/AgricultForest.62.4.24

\author{
Soghra KIANI and Kayvan AGAHI ${ }^{1}$
}

\title{
CORRELATION AND PATH COEFFICIENT ANALYSIS OF MORPHOLOGICAL TRAITS AFFECTING GRAIN SHAPE IN RICE GENOTYPES (ORYZA SATIVA L.)
}

\section{SUMMARY}

This work was undertaken to look into the interrelationships among morphological traits and rice grain shape. For this purpose, a set of 25 rice genotypes was sown and subjected to a farm survey, based on the standard evaluation system for rice. Correlation coefficient analysis showed that the grain shape was positively correlated with grain length, panicle length, plant height and the number of tillers while, there were statistically significant and negative correlations between grain shape with maturity date, number of grains per panicle, grain breadth, 100-grain weight and flag leaf width. Sequential path analysis revealed that grain breadth, grain length and number of grains per panicle, as first-order variables, was responsible for about $98 \%$ of the variation in grain shape. Also, 100-grain weight, maturity date, number of tillers and flag leaf width were determined as second-order predictors.

Amongst second-order predictors, 100-grain weight was a noteworthy trait regarding its high direct and indirect effects on grain breadth and grain length. Study of multicollinearity measures revealed that sequentializing of predictor variables reduced problems due to multicollinearity leading to a better understanding of the interrelationships among the various traits and their relative contribution. Also, the bootstrap analysis indicated that all direct effects were significant.

The results suggested that grain breadth, grain length and number of grains per panicle, as first-order predictor variables had the highest direct effect on grain shape and could be used as a selection criterion to improve rice grain shape. Also, 100-grain weight, maturity date, number of tillers and flag leaf width, as second-order predictor variables affect the rice grain shape indirectly through their effects on first-order predictors. The authors recommend for the use of sequential equation modeling to conduct a proper sequential path analysis.

Keywords:conventional path analysis, sequential path analysis, stepwise multiple linear regression, structural equation modeling

\footnotetext{
${ }^{1}$ Soghra Kiani , (corresponding author: so.kiani02@gmail.com), Department of Agriculture, Payam-e Noor University, Tehran, Iran, P. O. Box: 19395-3697, Kayvan Agahi, Department of Plant Breeding, College of Agriculture, Imam Khomeini International University, Qazvin, IRAN, P.O.Box: 34148-96818.

Notes: The authors declare that they have no conflicts of interest. Authorship Form signed online.
} 


\section{INTRODUCTION}

Rice appearance is a character considered as one of the main quality attributes by consumers therefore, measuring and understanding factors influencing rice grain appearance is a great challenge for industries and breeders in meeting consumer preferences (Haider et al., 2014). In genetics, grain shape has been widely accepted as a complex trait controlled by multiple genes with small effects (Yin et al., 2015). Thus, understanding the complexities governing the relationships among traits leads to increased selection gain in breeding programs. In this regard, correlation coefficients, multiple linear regression and path analysis are some of the common statistical methods. Determination of correlation coefficients is an important statistical procedure to examine the relationship between traits. However, increase of the number of independent variables controlling a particular dependent variable can lead to increased interdependence. In such situation, correlations may be insufficient to explain the associations in a way that will enable breeders to decide on a direct or indirect selection strategy (Ofori 1996; Sabaghnia et al., 2010).

Path coefficient analysis is a statistical tool that partitions correlation coefficients into direct effects (showing how a 1 unit change in the predictor variable will affect response variable) and indirect effects (association of one predictor variable with response variable mediated through other predictor variables in the model). It is obvious that decomposition of the effects depends on the model intended to describe the causal relationships among variables (modeling). Therefore, modeling is an important step towards path analysis.

Among path analyses, we can distinguish two types of models: (i) simple models, in which all traits except for the dependent one are set up at the same ontological level, which makes for their being treated as co-related; and (ii) complex models, in which traits are set up at different levels, and relations (that is, co-relations or cause-and-effect relationships) between them are to reflect possible biological relations. Complex models are referred to as se $q$ quential models reflecting sequential development of crop traits and, thus, sequentiality of cause-and-effect associations among them (Kozak and Azevedo 2014). Although traits are set up in some sequential order in the model, the methodology of this setting is based upon stepwise vari $\neg$ able selection in regression analysis and analysis of the total contriburtion of the traits to the variation of the dependent vari aable (Mohammadi et al., 2003). In a regression analysis however, collinearity (or multicollinearity) is the undesirable situation where the correlations among the independent variables are strong. Tolerance is a statistic used to determine how much the independent variables are linearly related to one another (multicollinear). Tolerance is the proportion of a variable's variance not accounted for by other independent variables in the model. A variable with very low tolerance contributes little information to a model, and can cause computational problems. VIF, or the variance inflation factor is the reciprocal of the tolerance. As the variance inflation factor increases, so does the variance of 
the regression coefficient, making it an unstable estimate. Large VIF values are an indicator of multicollinearity (IBM Released 2010).

The objective of this paper was to address the nature of the relationships between some prominent morphological traits and rice grain shape. The outcome could be helpful to develop selection strategies in genetic breeding programs aimed at improving marketability or cooking quality of rice.

\section{Plant materials and studied traits}

\section{MATERIAL AND METHODS}

Field experiment was carried out at the Tonekabon Rice Research Station located at $36^{\circ} 51^{\prime} \mathrm{N}, 50^{\circ} 46^{\prime} \mathrm{E}$ and -22 meter below the sea level. A set of twenty five rice genotypes was prepared from the Iran Rice Research Institute, Rasht including: Alfa, AliKazemi, Anbarboo, Binam, Century Patna231, Dom Siah, Gharib, Gharib Seyah Rayhani, Hassani, Hassan Saraei, Hassan Saraei Atashagah, Hassan Saraei Pichide Ghalaf, IR28, IR58, Lido, Line213, Line304, Line305, Mussa Tarom, Plano, Salari, Sanaderia, Sange Jo, Strella, and Zenith. After a primary growth in the nursery, the derived seedlings were transplanted to the main field, according to a squared lattice experimental design with two replications.

The mean of temperature and average annual rainfall of the station were $15 \mathrm{oC}$ and $1100 \mathrm{~mm}$, respectively. Each plot with an average area of $9 \mathrm{~m} 2$ comprised of four rows spaced $25 \mathrm{~cm}$ apart.

To collect data, rice plants were randomly selected and measured. Studied traits were: days to heading (DH); days to maturity (DM); plant height (PH); number of tillers (NT); panicle length (PL); flag leaf length (FL); flag leaf width $(\mathrm{FW})$; length of the uppermost inter-node (LU); grain length (GL); grain breadth (GB); hundred grain weight (GW); number of grains per panicle (GP). Grain shape (GS) was calculated as the ratio of GL to GB. All the traits were measured on the basis of the standard evaluation system for rice published by the International Rice Research Institute (IRRI 1996).

\section{Statistical analysis}

Normality of the distribution of the data was evaluated using skewness and kurtosis parameters, related to each trait, using AMOS 22 (AMOS 2013) statistical software. Simple correlation coefficients were calculated for all possible comparisons using Pearson correlation coefficient.

Stepwise multiple linear regression analysis was performed in order to determine the best sequential model representing causal relationship between predictor variables and rice grain shape. In fact, predictor variables were organized into first- and second-order paths. After identification of the model, direct and indirect effects of the predictor variables on response variable were computed using structural equation modeling (SEM) using AMOS 22 (AMOS 2013) statistical package. Correlation and regression analysis were carried out using IBM Statistics 19 (IBM Statistics 2010. To estimate the standard error of path coefficients, bootstrap analysis was performed using AMOS 22 (AMOS 2013) statistical package. 


\section{Simple correlations}

\section{RESULTS AND DISCUSSION}

Simple correlations and path coefficient analysis were studied to determine the interrelations governing direct and indirect grain shape components. The simple correlation coefficients (Table 1) showed there were significant correlations between grain shape and all of the measured traits except for FL, UL and DH. The correlation between grain shape and other traits was of great importance for us, because the main purpose of this study was to identify traits affecting the grain shape. As shown in Table 1, traits GL, PL, PH and NT were positively and significantly correlated with GS. There were statistically significant and negative correlations between GS and some other rice characters such as DM, GP, GB, GW and FW (Table 1).

Table 1. Pairwise Pearson correlation coefficients between 13 traits of 25 rice genotypes

\begin{tabular}{|c|c|c|c|c|c|c|c|c|c|c|c|c|}
\hline Trait & aH & DM & GP & GL & GB & UL & PL & PH & GW & NT & FW & FL \\
\hline DM & $0.72^{* *}$ & & & & & & & & & & & \\
\hline GP & 0.17 & 0.22 & & & & & & & & & & \\
\hline GL & 0.19 & $-0.34^{*}$ & $-0.46^{* *}$ & & & & & & & & & \\
\hline GB & -0.16 & 0.24 & $0.50^{* *}$ & $-0.70^{* *}$ & & & & & & & & \\
\hline UL & 0.22 & 0.22 & -0.03 & 0.01 & -0.01 & & & & & & & \\
\hline PL & $0.45^{* *}$ & 0.15 & $-0.41^{* *}$ & $0.42^{* *}$ & $-0.47^{* *}$ & $0.55^{* *}$ & & & & & & \\
\hline PH & $0.30^{*}$ & 0.06 & -0.17 & $0.39^{* *}$ & $-0.40^{* *}$ & $0.78^{* *}$ & $0.75^{* *}$ & & & & & \\
\hline GW & -0.18 & -0.20 & 0.25 & -0.06 & $0.57^{* *}$ & -0.06 & $-0.35^{*}$ & -0.24 & & & & \\
\hline NT & $0.35^{*}$ & 0.24 & $-0.41^{* *}$ & 0.12 & $-0.42^{* *}$ & -0.15 & $0.32^{*}$ & -0.01 & $-0.37 * *$ & & & \\
\hline FW & 0.17 & $0.35^{*}$ & $0.75^{* *}$ & $-0.48^{* *}$ & $0.49^{* *}$ & 0.05 & $-0.37^{* *}$ & -0.15 & 0.16 & -0.26 & & \\
\hline FL & $0.36^{* *}$ & $0.35^{*}$ & 0.19 & -0.22 & 0.18 & $0.38^{* *}$ & $0.41^{* *}$ & $0.31^{*}$ & 0.003 & 0.21 & $0.34^{*}$ & \\
\hline GS & 0.18 & $-0.32^{*}-0.46^{* *}$ & $0.87^{* *}$ & $-0.94^{* *}$ & 0.01 & $0.48^{* *}$ & $0.45^{* *}-0.41^{* *}$ & $0.29 *$ & $-0.49 * *$ & -0.20 \\
\hline
\end{tabular}
and ** mean that correlation is significant at the 0.05 and 0.01 level (2-tailed), respectively
The symbol of traits consist of: DH, days to heading; DM, days to maturity; GP, the number of grains per
panicle; GL, grain length; GB, grain breadth; UL, the length of the uppermost inter-node; PL, panicle length;
PH, plant height; GW, 100-grain weight; NT, the number of tillers; FW, flag leaf width; FL, flag leaf length;
GS, grain shape.

Considering that, grain length and grain breadth were the two important components of grain shape therefore, the relationship between grain length and grain breadth with other rice traits was also important. In this study, GL was significantly positively correlated with FW and FL while, it was significantly negatively correlated with DM and GP (Table 1). Significant positive correlations were observed between grain breadth with GP and FW while, the correlation between grain breadth with PL and NT was significantly negative (Table 1).

\section{Conventional and sequential path analysis}

To determine the relative importance of the traits affecting rice grain shape, the relevant correlation coefficients were separated into direct and indirect effects using conventional path analysis. 
Table 2. Direct (the bold numbers on the diagonal) and indirect effects of firstorder predictor variables on grain shape of 25 rice genotypes plus two common measures of collinearity (tolerance, Tol and variance inflation factor, VIF) in conventional path analysis

\begin{tabular}{|l|l|l|l|l|l|l|l|l|l|l|l|l|l|l|}
\hline Trait $^{\text {a }}$ & DH & DM & GP & GL & GB & UL & PL & PH & GW & NT & FW & FL & Tol & VIF \\
\hline DH & $\mathbf{- 0 . 0 4 3}$ & -0.031 & -0.007 & -0.008 & 0.007 & -0.009 & -0.019 & -0.013 & 0.008 & -0.015 & -0.007 & -0.015 & 0.07 & 15.25 \\
\hline DM & -0.006 & $-\mathbf{0 . 0 0 8}$ & -0.002 & 0.003 & -0.002 & -0.002 & -0.001 & -0.001 & 0.002 & -0.002 & -0.003 & -0.003 & 0.11 & 8.89 \\
\hline GP & 0.016 & 0.021 & $\mathbf{0 . 0 9 7}$ & -0.044 & 0.048 & -0.003 & -0.040 & -0.017 & 0.024 & -0.040 & 0.073 & 0.019 & 0.11 & 8.86 \\
\hline GL & 0.088 & -0.158 & -0.210 & $\mathbf{0 . 4 6 0}$ & -0.320 & 0.004 & 0.191 & 0.178 & -0.029 & 0.056 & -0.220 & -0.103 & 0.09 & 10.96 \\
\hline GB & 0.097 & -0.146 & -0.307 & 0.427 & $\mathbf{- 0 . 6 1 4}$ & 0.004 & 0.289 & 0.243 & -0.352 & 0.255 & -0.302 & -0.113 & 0.10 & 9.98 \\
\hline UL & -0.009 & -0.009 & 0.001 & 0.000 & 0.000 & $\mathbf{- 0 . 0 4 3}$ & -0.024 & -0.033 & 0.003 & 0.006 & -0.002 & -0.016 & 0.14 & 7.27 \\
\hline PL & -0.001 & 0.000 & 0.001 & -0.001 & 0.001 & -0.001 & $-\mathbf{0 . 0 0 1}$ & -0.001 & 0.000 & 0.000 & 0.001 & -0.001 & 0.09 & 11.55 \\
\hline PH & 0.021 & 0.004 & -0.012 & 0.027 & -0.027 & 0.053 & 0.051 & $\mathbf{0 . 0 6 9}$ & -0.016 & -0.001 & -0.010 & 0.021 & 0.10 & 9.69 \\
\hline GW & 0.008 & 0.009 & -0.011 & 0.003 & -0.026 & 0.003 & 0.016 & 0.011 & $\mathbf{- 0 . 0 4 5}$ & 0.016 & -0.007 & 0.000 & 0.22 & 4.63 \\
\hline NT & 0.001 & 0.000 & -0.001 & 0.000 & -0.001 & 0.000 & 0.001 & 0.000 & -0.001 & $\mathbf{0 . 0 0 2}$ & 0.000 & 0.000 & 0.16 & 6.16 \\
\hline FW & -0.003 & -0.007 & -0.015 & 0.010 & -0.010 & -0.001 & 0.007 & 0.003 & -0.003 & 0.005 & $\mathbf{- 0 . 0 2 0}$ & -0.007 & 0.10 & 9.85 \\
\hline FL & 0.006 & 0.006 & 0.003 & -0.004 & 0.003 & 0.006 & 0.007 & 0.005 & 0.000 & 0.003 & 0.006 & $\mathbf{0 . 0 1 6}$ & 0.24 & 4.23 \\
\hline
\end{tabular}

Dependent Variable: GS, Residual effect $=0.124$

${ }^{a}$ The symbol of traits consist of: DH, days to heading; DM, days to maturity; GP, the number of grains per panicle; GL, grain length; GB, grain breadth; UL, the length of the uppermost inter-node; PL, panicle length; PH, plant height; GW, 100-grain weight; NT, the number of tillers; FW, flag leaf width; FL, flag leaf length; GS, grain shape.

The results concerning direct and indirect effects of the studied traits on rice grain shape using conventional path analysis, where all traits were considered as first-order variables, with grain shape as the response variable (Model I), are presented in Table 2. Also, the results of two measures of collinearity analysis (Tolerance and Variance Inflation Factor) are presented in this Table.

As shown in Table 2, according to the conventional path analysis and collinearity analysis, the collinearity measure (VIF) was almost high for some traits such as $\mathrm{DH}(\mathrm{VIF}=15.25), \mathrm{PL}(\mathrm{VIF}=11.55)$ and $\mathrm{GL}(\mathrm{VIF}=10.98)$. Also, is some cases the VIFs were close to $10(\mathrm{~GB}, \mathrm{VIF}=0.98$; $\mathrm{PH}, \mathrm{VIF}=9.69 ; \mathrm{FW}$, $\mathrm{VIF}=9.85$ ) suggesting the potential existence of multicollinearity.Results of the sequential path analysis are shown in Table 3, where predictor traits, as grouped into first- and second-order variables, and grain shape as the response variable (Model II). Compared with the model I, grouping predictor variables by means of stepwise multiple linear regression resulted in decreased VIF values for all predictors (Table 3). Thus, compared with model I, application of sequential path analysis facilitated detection of the actual contribution of predictors with minor confounding effects. Likewise, some researchers such as Mohammadi et al., (2003) reported that, sequential path procedure reduces collinearity problems leading to a better understanding of the interrelationships among the various traits and their relative contribution.

Normally, researchers are interested in testing the significance of statistics obtained in various statistical analyses. To perform such tests, standard error of the statistic is needed. 
Table 3. Direct effects of predictor variables on the grain shape of 25 rice genotypes along with two common measures of collinearity values (tolerance and variance inflation factor, VIF) for predictor variables in conventional path analysis (CPA, all predictor variables as first-order variables) and sequential path analysis (SPA, predictors grouped into first- and second-order variables)

\begin{tabular}{|c|c|c|c|c|c|c|c|c|c|}
\hline \multirow{2}{*}{$\begin{array}{c}\text { Predictor } \\
\text { variables }\end{array}$} & \multirow{2}{*}{$\begin{array}{c}\text { Response } \\
\text { variable }\end{array}$} & \multicolumn{2}{|c|}{ Direct Effect } & \multicolumn{2}{c|}{ Tolerance } & \multicolumn{2}{|c|}{ VIF } \\
\hline & SPA & CPA & & SPA & CPA & SPA & CPA \\
\hline GB & GS & -0.66 & -0.61 & & 0.48 & 0.10 & 2.10 & 9.98 \\
\hline GL & & 0.43 & 0.46 & & 0.50 & 0.09 & 1.99 & 10.96 \\
\hline GP & & 0.07 & 0.10 & & 0.73 & 0.11 & 1.37 & 8.86 \\
\hline GL & GB & -0.21 & -0.43 & & 0.83 & 0.12 & 1.20 & 8.42 \\
\hline GW & & 0.53 & 0.46 & & 0.85 & 0.38 & 1.18 & 2.64 \\
\hline NT & & -0.28 & -0.25 & & 0.81 & 0.20 & 1.24 & 5.08 \\
\hline DM & & 0.33 & 0.27 & & 0.79 & 0.12 & 1.27 & 8.43 \\
\hline GB & GL & -0.84 & -0.47 & & 0.67 & 0.13 & 1.49 & 7.66 \\
\hline GW & & 0.42 & 0.26 & & 0.67 & 0.23 & 1.49 & 4.36 \\
\hline FW & GP & 0.69 & 0.39 & & 0.93 & 0.13 & & 1.07 & 7.96 \\
\hline NT & & -0.23 & -0.33 & & 0.93 & 0.18 & 1.07 & 5.58 \\
\hline
\end{tabular}

The symbol of traits consist of: DM, days to maturity; FW, flag leaf width; GB, grain breadth; GL, grain length; GP, the number of grains per panicle; ; GS, grain shape; GW, 100-grain weight; NT, the number of tillers.

Bootstrap analysis, as a re-sampling technique, provides an estimation of the standard error of any statistic. Results of the bootstrap analysis for direct effects are presented in Table 4. The low standard error and bias for all the path coefficients indicated the robustness of the model II in comparison with the model I.

Table 4. Estimation of standard error values of path coefficients using bootstrap analysis with 200 bootstrap samples

\begin{tabular}{|c|c|c|c|c|c|c|c|}
\hline $\begin{array}{c}\text { Predictor } \\
\text { variables }\end{array}$ & \multirow{2}{*}{$\begin{array}{c}\text { Response } \\
\text { variable }\end{array}$} & \multirow{2}{*}{ Adj. $\mathrm{R}^{2}$} & \multirow{2}{*}{$\begin{array}{l}\text { Direct } \\
\text { Effect }\end{array}$} & & \multicolumn{3}{|c|}{ Bootstrap } \\
\hline GB & GS & 0.98 & -0.662 & & -0.666 & -0.004 & 0.029 \\
\hline GL & & & 0.430 & 0.429 & -0.001 & 0.026 \\
\hline GP & & & 0.071 & & 0.066 & -0.005 & 0.037 \\
\hline DM & GB & 0.71 & 0.333 & & 0.333 & 0.000 & 0.079 \\
\hline GL & & & -0.214 & -0.213 & 0.001 & 0.069 \\
\hline GW & & & 0.525 & & 0.526 & 0.001 & 0.081 \\
\hline NT & & & -0.275 & -0.275 & 0.000 & 0.067 \\
\hline GB & GL & 0.64 & -0.840 & -0.84 & 0.000 & 0.119 \\
\hline GW & & & 0.418 & 0.425 & 0.007 & 0.129 \\
\hline FW & GP & 0.62 & 0.694 & & 0.692 & -0.002 & 0.075 \\
\hline NT & & & -0.232 & -0.243 & -0.011 & 0.100 \\
\hline
\end{tabular}

The symbol of traits consist of: DM, days to maturity; FW, flag leaf width; GB, grain breadth; GL, grain length; GP, the number of grains per panicle; ; GS, grain shape; GW, 100-grain weight; NT, the number of tillers. Calculation were carried out by usin AMOS statistical pakage (AMOS 2013).

Graphical presentation of the results facilitates understanding of the complex relationship among the various variables and their contributions. In this regard, the diagram of sequential path analysis drawn by using AMOS statistical package helps in better understanding of the complexity of relationships between 
variables (Figure 1). The Root Mean Square Error of Approximation (RMSEA) fit statistic for the model was 0.091 .

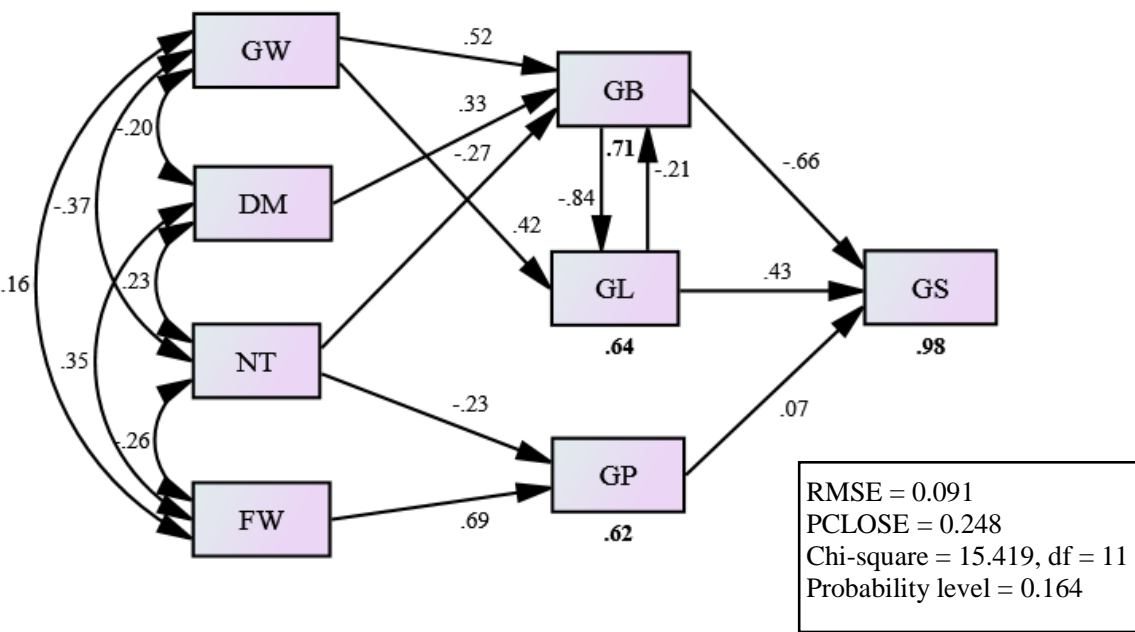

The diagram was drawn using AMOS statistical package (AMOS 2013). The adjusted R square values have been indicated by bold numbers below each of the dependent variables.

The symbol of traits consist of: DM, days to maturity; FW, flag leaf width; GB, grain breadth; GL, grain length; GP, the number of grains per panicle; ; GS, grain shape; GW, 100-grain weight; NT, the number of tillers.

Figure 1. Causal model diagram illustrating the interrelationships among various traits contributing to rice grain shape.

The PCLOSE represents the $p$ value for testing the null hypothesis that the population RMSEA is no greater than 0.05 indicating a close fit of the model in relation to the degrees of freedom. Also, the Chi-square (CMIN statistic) value was equal to 15.42 . The $p$ value was 0.164 revealing a proper goodness of fit for the model.

Estimation of the adjusted coefficient of determination (Adj. $\mathrm{R}^{2}$ ) revealed that GB, GL and GP as first-order variables accounted for about $98 \%$ of the variation in grain shape (Figure 1 and Table 4). Also, the results of sequential path analysis, when the second-order variables were used as predictors, and the first-order variables as response variables, indicated that GW, DM positively and GL, NT negatively influenced the GB and accounted for $71 \%$ of the observed variation. Moreover, the GW positively and the GB negatively influenced GL and accounted for $64 \%$ of the observed variation while, FW positively and NT negatively influenced the GP and accounted for $62 \%$ of the observed variation (Figure 1 and Table 4). Direct and indirect effects of first- and second-order predictor variables on response variables, including GB, GL, GP and GS have been presented in Table 5. According to this table, the indirect effects of DM and NT on GS were low while, indirect effects of FW, GW, GL and GB on GS were 
high. Amongst second-order predictors, GW was a noteworthy trait with regard to its high direct and indirect effects on GB and GL. Also, NT had a negative direct effect on GP while, FW showed a relatively high positive direct effect on GP. As shown in Table 5, in this study, one of the remarkable advantages of using SEM was that the indirect effects resulting from second-order predictors on GS were also estimated.

Table 5. Direct (d) and indirect (i) effects for the predictor variables in sequential path analysis.

\begin{tabular}{|c|c|c|c|c|c|c|c|c|}
\hline \multirow{2}{*}{ Response variable } & & \multicolumn{2}{|c|}{ predictor variables } \\
\cline { 2 - 10 } & & FW & DM & NT & GW & GL & GB & GP \\
\hline GL & d & - & - & - & 0.418 & - & -0.84 & - \\
\hline & i & - & -0.064 & 0.033 & -0.933 & 0.219 & -0.322 & - \\
\hline GB & d & - & 0.333 & -0.275 & 0.525 & -0.214 & - & - \\
\hline & i & - & 0.008 & -0.004 & 0.007 & -0.027 & 0.219 & - \\
\hline GP & d & 0.694 & - & -0.232 & - & - & - & - \\
\hline & i & - & - & - & - & - & - & - \\
\hline GS & d & - & - & - & - & 0.43 & -0.662 & 0.071 \\
\hline & i & 0.217 & -0.071 & 0.035 & -0.692 & 0.243 & -0.931 & - \\
\hline
\end{tabular}

The symbol of traits consist of: DM, days to maturity; FW, flag leaf width; GB, grain breadth; GL, grain length; GP, the number of grains per panicle; ; GS, grain shape; GW, 100-grain weight; NT, the number of tillers.

Calculation were carried out by usin AMOS statistical pakage (AMOS 2013).

Identifying and analysis of associations between quantitative traits is important because, it can be used as a resource for designing selection-based breeding programs in the future. Most of the correlations obtained in this study were consistent with other studies. For example, likewise, significant positive correlations $(P<0.01)$ have been reported by Rabiei et al., $(2004)$ between: GL and GS, GB and GW, GS and PH, DH and DM, DH and PL, PH and PL. Moreover, they reported significant negative correlations $(P<0.01)$ between: GL and GB, GB and GS, GB and PH, GB and PL which were totally in agreement with the results of the present work. Furthermore, in contrast to our results, they reported a significant positive correlation between PL and GP. Similar to the results of current work, a significant positive correlation between PH and PL was found by Hossain et al., (2015) and Kishore et al., (2015) while, Rai et al., (2015) reported it significant, but negative. According to our observations and in agreement with Hossain et al., (2015), the correlation between GW and GP was significant and positive while, Kishore et al., (2015) reported it significantly negative. A significant positive correlation between GW and GL was reported by Haider et al., (2014) and Kumar (2015) while, like current study, Rafii et al., (2014) found no such correlation. Similar to the results of this study, Hossain et al., (2015) found a significant negative correlation between NT and GP. They also reported a positive correlation between NT and DM while was in contrast to our observations. It seems that the discrepancies between the reports, regarding correlation coefficients between traits, are largely caused by working with 
different populations, regarding the fact that diverse populations also have a different genetic structure. In this study, some relatively strong correlations were recorded such as FW-GP, DH-DM and PH-UL .Strong correlations are likely due to pleiotropy or linkage. QTL mapping is a method to answer whether linkage or pleiotropic effect is the responsible for the occurrence of a strong correlation (Chen and Lübberstedt 2010).

Previous researches have indicated that path analysis provides more information on the interrelationships between traits than correlation coefficients (Kozak and Kang 2006; Sabaghnia et al., 2010). Path analysis provides a framework for better understanding of how plant morphological components affect a final target trait in field crops through determination of the most important factors that directly and/or indirectly affect the final target trait.

In path coefficient analysis the amount that a trait contributes to yield is influenced by the different traits through different paths. Imprecise assessment of a trait's contribution through incorrect pathways may misdirect breeding attempts, thus limiting the efficiency in selecting favorable cultivars (Agrama 1996; Sabaghnia et al., 2010). Also the conventional approach for path analysis might result in a multicollinearity of variables, particularly when the correlations among some of the variables are high (Mohammadi et al., 2003). On the other hand, yield and shape-related traits are complicatedly interrelated, often leading to high multicollinearity. Thus, in this study, sequential path analysis was used to avoid the problems due to conventional path analysis and multicollinearity of variables. Such a strategy has been used before by researchers (Mohammadi et al., 2003; Sabaghnia et al., 2010).

Most of the studies on the path coefficient analysis in rice have been conducted to analyze yield and yield components. Rabiei et al., (2004) performed sequential path analysis to identify direct and indirect effects of traits affecting GS. Based on their results, GB, GL and PH were determined as first-order predictor variables whereas, GE, PL and GP were as second-order predictors. In the present study however, GP included in the path model as first-order variable rather than PH whereas, GW, DM, NT and FW were considered as second-order predictors. In this study, the adj. $\mathrm{R}^{2}$ value calculated for each dependent variable was more than that of reported by (Rabiei et al., (2004)).

As mentioned by Kozak and Azevedo (2014), path analysis is an integral part of structural equation modeling. Despite of this, some researchers use standardized stepwise multiple linear regression to organize the variables into different order paths to determine causal relationships. Subsequently, they draw the path diagram manually by using common software such as MS office Word. In fact, to construct the path diagram, they integrate information obtained from each separate regression analysis while the standardized regression coefficients are used as the path coefficients. Such a method of demonstration of information may be accompanied with error because of the following reasons: 
-By employing such a method, we assume that all of the model variables are perfectly measured (i.e. no measurement error). Hence, the ignored errors will downward bias of parameter estimates and leads to non-sign

-Normally, the model is structural when several paths are analyzed simultaneously. Therefore, inter-relationships among variables in the model would be complex. For example, in a sequential path diagram (similar to Figure 1) the first-order variables play two fully featured roles simultaneously as: (i) each of them acts as a response variable for the second-order predictors and (ii) they play the role of predictor variables for the main response variable. We don't see such a complexities in separate regression analyses.

-It is natural that there may be reciprocal effects between first-order predictor variables (see Figure 1) while, stepwise regression analysis can't estimate such effects because they belong to separate models and stepwise regression analysis can't integrate them into one model.

Therefore, it is always useful to switch to a full SEM using special software designed for this purpose such as AMOS. Although the process through which AMOS calculates the path coefficients is similar to a standardized regression, however, when we make the model sequential, (i.e. when we have both first- and second-order predictor variables in the model at the same time) the AMOS calculates path coefficients using a more complicated model. Hence, the outcome can't be equal as compared to the separate standardized regression coefficients.

\section{REFERENCES}

Agrama HAS.1996. Sequential path analysis of grain yield and its components in maize. Plant Breeding 115:343-346

Chen Y and Lübberstedt T.2010. Molecular basis of trait correlations. Trends Plant Sci 15:454-461

Haider Z, Mehboob A, Razaq A, Khalid U, Rasool N and Mehmood K.2014. Effect of drought stress on some grain quality traits in rice (Oryza sativa L.). Academia Journal of Agricultural Research 2:128-133

Hossain S, Maksudul Haque M and Rahman J.2015. Genetic Variability, Correlation and Path Coefficient Analysis of Morphological Traits in some Extinct Local Aman Rice (Oryza sativa L). Journal of Rice Research 3:2

IBM.Released 2010. IBM SPSS Statistics for Windows, Version 19. In. IBM Corp, Armonk, NY

IRRI.1996. Standard evaluation system for rice. 4th ed. International Rice Research Institute, Manila, Philippines

Kishore N, Srinivas T, Nagabhushanam U, Pallavi M and Sameera S.2015. Genetic variability, correlation and path analysis for yield and yield components in promising rice (Oryza sativa L.) genotypes. SAARC Journal of Agriculture 13:99-108

Kozak M and Azevedo RA.2014. Sequential path analysis: what does" sequential" mean? Scientia Agricola 71:525-527 
Kozak M and Kang MS.2006. Note on modern path analysis in application to crop science. Communications in Biometry and Crop Science 1:32-34

Kumar V.2015. Variability and correlation studies for grain physicochemical characteristics of rice (Oryza sativa L.). The Bioscience 10:917-922

Mohammadi S, Prasanna B and Singh N.2003. Sequential path model for determining interrelationships among grain yield and related characters in maize. Crop Sci 43:1690-1697

Ofori I.1996. Correlation and path-coefficient analysis of components of seed yield in bambara groundnut (Vigna subterranea). Euphytica 91:103-107

Price TD, Qvarnström A and Irwin DE.2003. The role of phenotypic plasticity in driving genetic evolution. Proceedings of the Royal Society of London B: Biological Sciences 270:1433-1440

Rabiei B, Valizadeh M, Ghareyazie B and Moghaddam M.2004. Evaluation of selection indices for improving rice grain shape. Field Crops Res 89:359-367

Rafii M, Zakiah M, Asfaliza R, Haifaa I, Latif M and Malek M.2014. Grain quality performance and heritability estimation in selected F1 rice genotypes. Sains Malaysiana 43:1-7

Rai P, Sarker U, Roy P and Islam A.2015. Character association in $\mathrm{f} 4$ generation of rice (Oryza sativa L.). Bangladesh Journal of Plant Breeding and Genetics 26:39-44

Sabaghnia N, Dehghani H, Alizadeh B and Mohghaddam M.2010. Interrelationships between seed yield and 20 related traits of 49 canola (Brassica napus L.) genotypes in non-stressed and water-stressed environments. Spanish Journal of Agricultural Research 8:356-370

Yin C, Li H, Li S, Xu L, Zhao Z and Wang J.2015. Genetic dissection on rice grain shape by the two-dimensional image analysis in one japonicax indica population consisting of recombinant inbred lines. Theor Appl Genet 128:1969-1986 